\section{ETNOARQUEOLOGIA APLICADA A LA METALURGIA DEL ORO: EL CASO DE EUROPA ATLANTICA Y AFRICA OCCIDENTAL (1)}

ETHNOARCHAEOLOGY APPLIED TO

GOLD METALLURGY: THE CASE OF

ATLANTIC EUROPE AND WEST AFRICA

\section{BARBARA REGINA ARMBRUSTER (*)}

\section{RESUMEN}

La tecnología del oro durante el Bronce Atlántico se explica a la luz de la investigación etno-arqueológica y experimental. Los fulbés, un pueblo seminómada de Malí, conservan una antigua y rica tradición en la producción de orfebrería que puede tomarse como fuente de procesos técnicos antiguos, hoy ya desaparecidos en nuestro entorno europeo. La autora defiende el empleo del método experimental en arqueología, además de la validez de las analogías funcionales y etnológicas para la reinterpretación de explicaciones tecnológicas incorrectas que se han dado por supuestas en demasiadas ocasiones.

\begin{abstract}
The technology of Atlantic Bronze Age gold-working is studied in the light of ethno-archaeological and experimental research. The Fulbes, a semi-nomadic group in Mali, retain an old and rich tradition of gold jewellery production that displays techniques and processes which have disappeared in Europe. The author defends the experimental method in archaeology, and uses ethnological and functional analogies to reinterpret incorrect technological assumptions that all too often have been taken for granted.
\end{abstract}

(1) La traducción del original alemán ha sido corregida por Alicia Perea y revisada por la autora. Las fotografías son de B. Armbruster y los dibujos de G. Wittner y B. Armbruster.

(*) Seminar für Vor- und Frühgeschichte. Frankfurt Universität. Frankfurt.
Palabras clave: Orfebrería. Tecnología. Bronce Atántico europeo. Metalurgia. Minería. Etnología. Africa Occidental. Mali. Fulbés.

Key words: Goldwork. Technology. Atlantic Bronze Age. Metallurgy. Mining. Ethnology. Western Africa. Malí. Fulbés.

Malí es un país del Africa Occidental (Fig. 1) con una rica tradición, que ha producido una orfebrería única en su género. Aún hoy se puede seguir aquí todo el circuito de la elaboración de metales preciosos con métodos antiguos: desde la extracción del oro en ricas y productivas minas, pasando por la producción de herramientas de orfebre hasta llegar a la fabricación de ornamentos en los talleres orfebres.

Entre los numerosos trabajos de orfebrería, resultan de especial interés en los aspectos estético, técnico e histórico las joyas de los fulbés, un pueblo seminómada y ganadero en el delta interior del río Níger. Algunas piezas de oro, estilística y técnicamente comparables, se conocían ya en Europa Occidental durante la Edad del Bronce. También son comparables con los aros más recientes del Africa Occidental los collares torsionados en bronce de la Edad 


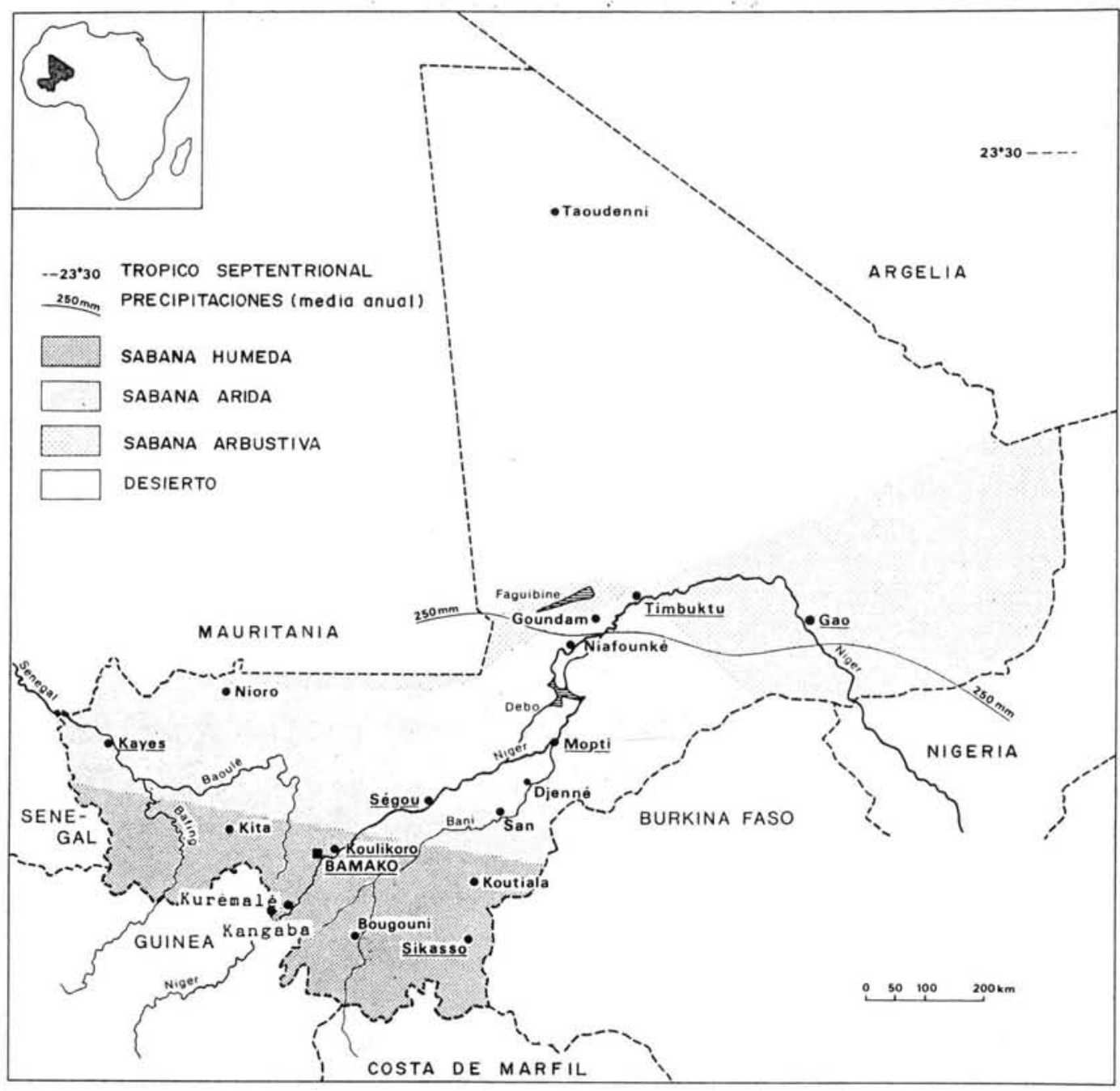

Fig. 1. Mapa esquemático del área estudiada del Africa occidental (según Gardi, 1988: 8).

del Hierro Antiguo en Europa Central y Septentrional. Mediante una investigación etnoarqueológica se ha podido reconstruir la fabricación de estos ornamentos (2). La analogía en la técnica artesanal sirve como modelo explicativo

(2) Los aspectos de la metalurgia tradicional aquí descritos fueron estudiados durante varias estancias de la autora (1986/87/88) en Mali. Aprendí el oficio con los orfebres y trabajé con herreros, mineros y fundidores de bronce. El tema fue objeto de investigación de un estudio detallado sobre recientes técnicas metalúrgicas en Africa Occidental (Armbruster y Dillhöfer, 1988) y sobre técnicas de los metalúrgicos prehistóricos (Armbruster, 1989, con amplios datos bibliográficos, pp. 191-210). Tenemos que limitarnos aquí a un breve resumen de los resultados esenciales de estas observaciones. para comprender mejor las técnicas de la orfebrería prehistórica, de la que sólo conocemos sus productos.

\section{LOS AROS DE ORO TORSIONADOS DE LA EDAD DE BRONCE}

Los aros de oro torsionados y martillados se conocen en la Edad del Bronce en el espacio atlántico: de las Islas Británicas (Fig. 2B) (Eogan, 1964: 272-283, 1967; Taylor, 1980: 3638), de Francia (Eluére, 1982: 48-51, 176-178; Briard 1990: 135-136) y de la Península Ibérica (Fig. 2.1) (Parreira y Pinto, 1980: 16, n⿳ำ 100; Armbruster, en preparación). 


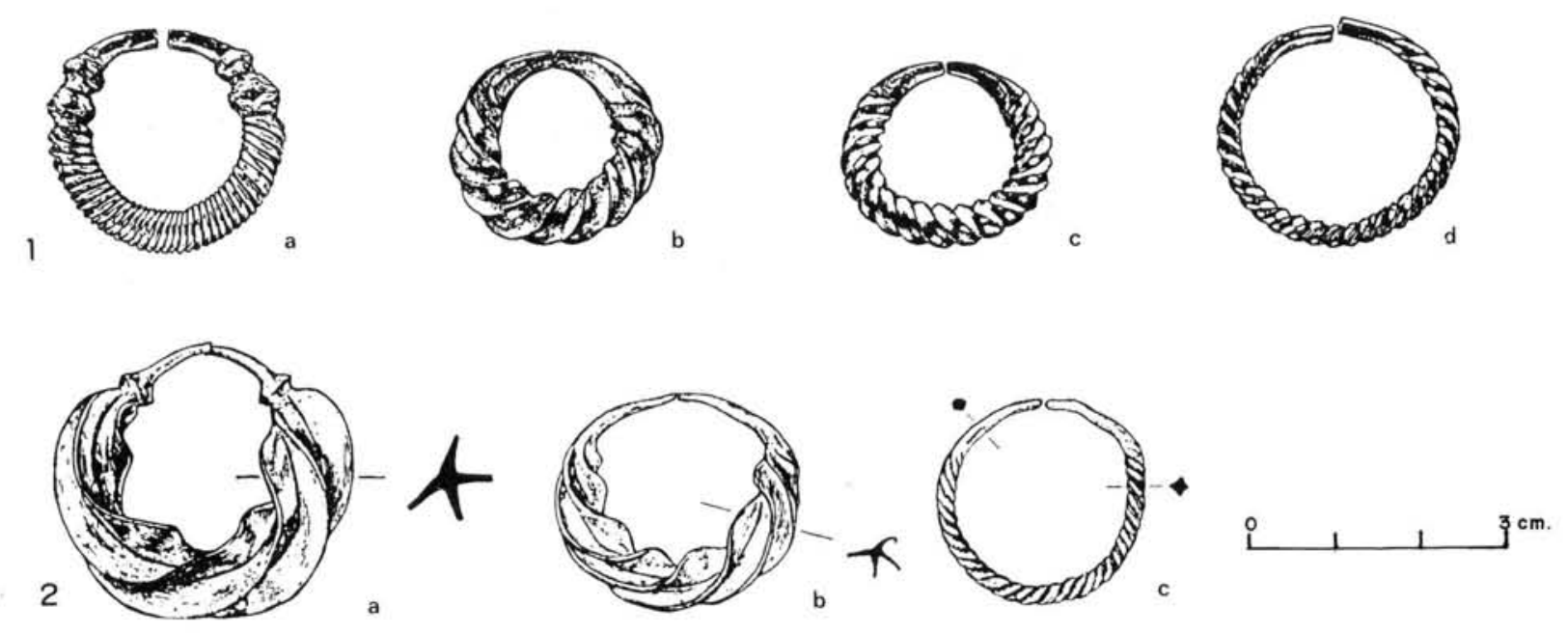

Fig. 2. 1) Aros de oro a partir de una barra doblada, sin indicación del lugar de hallazgo del Museu Nacional de Arqueologia Leite de Vasconcelos (Lisboa): a) $\mathrm{n}^{\circ}$ inv. 54; b) $\mathrm{n}^{\circ}$ inv. 56; c) $\mathrm{n}^{\circ}$ inv. 55; d) $\mathrm{n}^{\circ}$ inv. 53 .

2) Pendientes de oro irlandeses hacia 1200 a.d.C. (según Hawkes. 1961: Fig. 2): a) Castlereagh, Co. Roscommon; b) Co. Meath; c) sin indicación del lugar de hallazgo.

Se conocen asimismo piezas semejantes del Mediterráneo oriental, si bien pertenecen cronológicamente a una fase anterior y fueron fabricadas de otro modo (Mayron, 1936: 3-5; Hawkes, 1961: fig. 2). Recientemente, se ha vuelto a relacionar, sin tener en cuenta el diferente modo de fabricación, las piezas «striptwisted" de Gaza con los ejemplares "flangetwisted" de Castlereagh, Co. Roscommon (Fig. 2.2) (Briard, 1990: 134-135, fig. 7; Eogan, 1990: 157-159, fig. 3).

Las joyas torsionadas se presentan en formas, tamaños y secciones diferentes. Se interpretan como adornos para la cintura, el cuello o las orejas (Armstrong, 1920: torques, 20.21, Pl. 12.; pendientes, 38, Pl. 18 415-422; Eogan, 1983: cintura, Pl. 13). La sección del cuerpo del aro y el acabado de sus extremos responden al empleo de dos técnicas determinadas, respectivamente. Todas estas joyas tienen - como sus predecesoras de bronce (Evans, 1881: Figs. 466469) —, forma de espiral.

Las formas macizas, para cuya fabricación se requieren considerables cantidades de oro, no se pueden conectar fácilmente con la tradición de los trabajos orfebres en chapa, tales como lúnulas o soles, de la Edad del Bronce Antiguo. Su aparición en las Islas Británicas se interpreta como una reacción a la influencia continental procedente del Norte de Europa, la cual incitó a los orfebres a romper con su «linear geometric sheet style» de la Edad del Bronce Antiguo y a desarrollar formas propias con piezas macizas importadas (Taylor, 1980: 60; Catálogo de Colonia, 1983: 1). Dado que los hallazgos de piezas de oro de esta época en las Islas Británicas son especialmente numerosos, y para su fabricación eran necesarias considerables cantidades de oro, se sospecha que se habían abierto nuevos filones, o que se habían desarrollado mejores métodos de extracción.

Los aros de oro de Francia noroccidental y occidental se ponen en relación con los torques de las Islas Británicas (Taylor, 1980: 62). El hallazgo de Francia suroccidental (Carcassone, Departament del Aude) (Fig. 3) apunta, segun C. Hawkes (1961), a una conexión con el Mediterráneo. La orfebrería europea occidental de finales del segundo milenio antes de Cristo se caracteriza por los aros con torsión y el abandono de la decoración ornamental; sin embargo, no dejan de aparecer también otras formas 


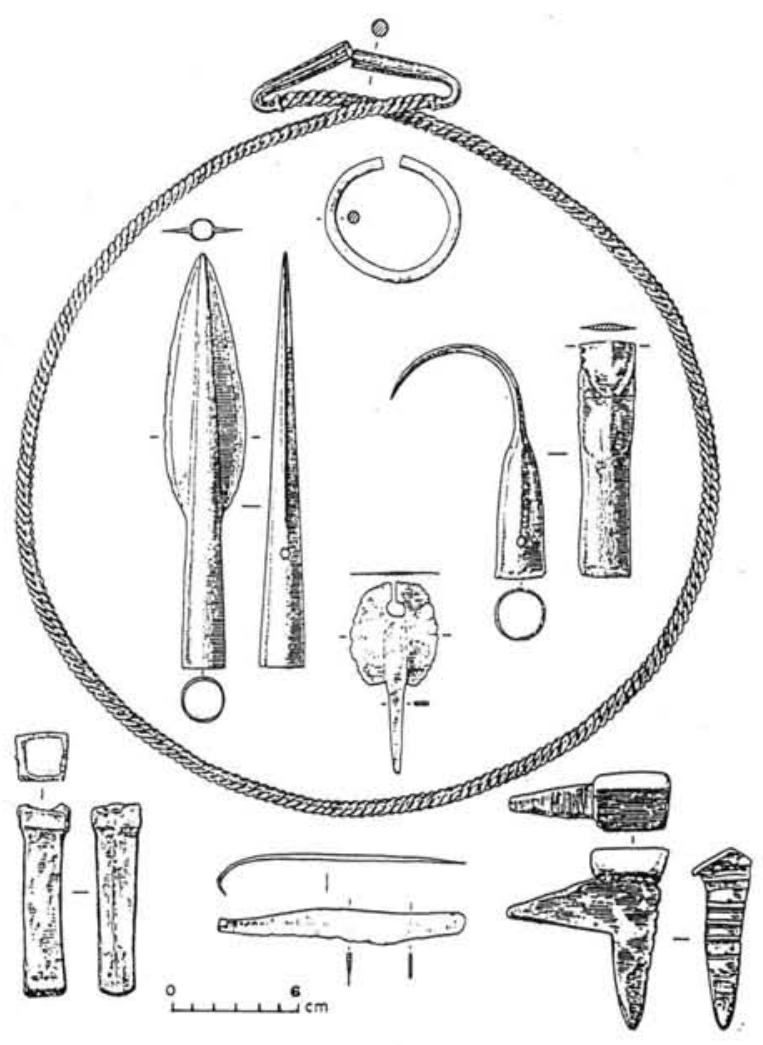

Fig. 3. Depósito de Fresné-la-Mère (según Eogan, 1967: Fig. 8).

ornamentales en oro, tales como anillos compuestos, pequeñas láminas lisas, brazaletes laminares lisos o estriados, pulseras abiertas, sin adornar, de sección redonda o romboidal (Eogan, 1964: 279, fig. 6; Taylor, 1980: tab. 30; Eluère, 1982).

A comienzos de la Edad del Hierro, aparecen collares de bronce de vueltas alternas: los "wendelringe». Cronológicamente, son más recientes que las joyas de oro torsionadas, y su ámbito de expansión abarca Europa Central y Septentrional. Aparecen tipos muy numerosos, con secciones y cierre de los extremos diferentes (Pietzsch, 1964; Heynowski, 1992: 16-27). La técnica de fabricación de estos collares de bronce en espiral de sección cruciforme con lóbulos muy pronunciados y de aros de oro a partir de una barra torsionada con perfil cruciforme es la misma (Armbruster, 1989: 135-161). Fueron martillados a partir de una pieza por conformado $\sin$ arranque de virutas y sin soldadura.

\section{LAS HERRAMIENTAS DE ORFEBRE DEL BRONCE FINAL}

Entre los hallazgos de la Edad del Bronce, aparecen menos herramientas para trabajar el metal que adornos fabricados con ellas. Lamentablemente, los hallazgos de herramientas de los primeros metalúrgicos se conocen sólo en unos pocos depósitos, en hallazgos aislados; y prácticamente nada procedente de asentamientos o tumbas (3). Hay dos depósitos, sin embargo, de los que se conocen utensilios de artesanos del metal que podrían haber servido para la fabricación de aros torsionados. En el depósito de Fresné-la-Mere, Departamento de Calvados (Eogan, 1967: fig. 8) se han asociado un torques de sección cruciforme y una pulsera de oro con varios objetos de bronce, dos de los cuales podrían interpretarse como el martillo y el yunque de un orfebre (Fig. 3). En el depósito de herramientas irlandés de Bishopsland, Condado de Kildare (Eogan, 1964: fig. 5), encontramos un yunque, tres martillos de cubo, dos cinceles, un punzón y una virola (Fig. 4). Cronológicamente, estos depósitos pertenecen, al igual que los aros de oro torsionados, al final de la Edad del Bronce. Los yunques de ambos depósitos pueden compararse tipológicamente con los utensịlios de los orfebres malíes, ya que presentan un ángulo de salida menor de $90^{\circ}$

\section{HIPOTESIS SOBRE LA TECNICA DE FABRICACION}

Muchos autores han escrito sobre la fabricación de los aros torsionados de sección cruciforme, pero pocos han comprendido el proceso (4). Entre las propuestas se presentan gran cantidad de opiniones que describen los siguientes modos de fabricación: vaciado por el método

(3) Sobre las herramientas prehistóricas de orfebres, véase sobre todo: Ohlhaver, 1939; Nicolardot y Gaucher, 1975; Ehrenberg, 1981; Jockenhövel, 1982; Mohen, 1984/85.

(4) Diferentes hipótesis acerca de la técnica de producción de los torques de bronce y oro, y aros en espiral con sección cruciforme en: Cohausen, 1881: 176ss.; Baldes, 1905: 46ss.; Bruckmann, 1914: 36, fig. 19; Goetze, 1925: 172; Maryon, 1936: 5-23; Krone, 1937: 221; Maryon, 1936: 205-209; Oldeberg, 1943: 110; Brunn, 1953: 10-13; Pietzsch, 1964; Taylor, 1980: 10-13; Untracht, 1982: 251, fig. 6-257; Tylecote, 1986: 104. 


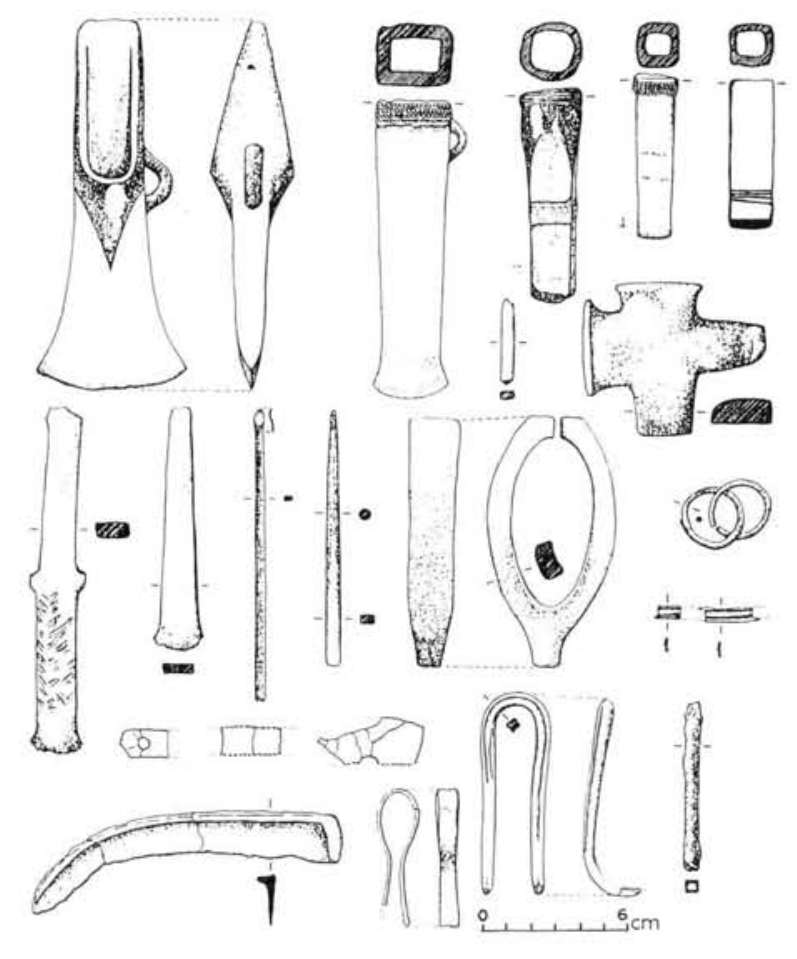

Fig. 4. Depósito de Bishopsland. Co. Kildare (según Eogan, 1983: Fig. 10).

de fundido a la cera perdida, soldadura, estañadura, talla, trefilado con una hilera especial, golpeado con estampilla, hasta, de hecho, la fabricación por martillado. Mediante la colaboración entre arqueólogos y técnicos se han logrado resultados aceptables. Los únicos experimentos sistemáticos y acertados los emprendió M. Pietsch (1964), aunque ya V. Cohausen (1885/86: 176-177) había descrito la fabricación de los «wendelringe» como trabajo de martillo.

\section{LA ANALOGIA ETNOARQUEOLOGICA: ORFEBRERIA EN MALI (AFRICA OCCIDENTAL)}

«L'etranger ne voit ce qu'il sait».

Proverbio africano (Anquetil, 1977: 10)

Todavía hoy, las joyas torsionadas comparables morfológica y técnicamente con las de la Edad del Bronce (Zeltner, 1931: 45. Eluère, $1980 / 81$ ) forman parte del vestido de las mujeres del Africa Occidental. En Malí resulta posible todavía seguir los diferentes aspectos de la orfebrería tradicional: extracción de oro, talleres de orfebrería, herramientas, técnicas, simbolismo y el modo de llevar los aros de oro, así como también sus imitaciones.

\section{LA MINERIA DE ORO TRADICIONAL EN MALI (ARMBRUSTER, 1992)}

Los buscadores de oro en la zona fronteriza entre Malí y Guinea Conakry viven a unos 10 $\mathrm{km}$ del yacimiento (Fig. 1, los poblados Korémalé y Kangaba). Los ricos yacimientos aluviales de oro se hallan en Bure, cuenca legendaria, descrita en fuentes de la Edad Media y explotada desde hace siglos (Levtzion, 1973: 17f. 155; Kiethega, 1983).

En cada "campo de oro" hay un herrero ambulante que fabrica herramientas para los mineros. Su fragua móvil contiene un fuelle manual de doble tubo, el yunque sujeto a una traviesa de madera, cincel, tenazas y varios martillos. El herrero no sólo trabaja aquí el hierro, sino también madera para los mangos de las piquetas y azadas, así como cáscaras de calabazas - recipientes indispensables para el transporte y lavado de la tierra aurífera. Cada tarde, el herrero lleva consigo sus herramientas en una bolsa o una caja pequeña. $\mathrm{Al}$ arqueólogo no le quedará nada que encontrar.

Trabajando en equipo, un grupo de buscadores cava pozos verticales de unos $80 \mathrm{~cm}$. de diámetro y $18-10 \mathrm{~m}$. de profundidad en el suelo estéril hasta el estrato aurífero del yacimiento aluvial. De esta manera, se cavan con una distancia de aproximadamente $1,5 \mathrm{~m}$. varios pozos en fila, que pueden seguir el estrato aurífero durante algunos kilómetros. Los pozos se comunican por medio de una galería situada a la altura del aluvión (Fig. 5). El pozo lo perforan generalmente 2 o 3 mineros. Uno cava con una piqueta y una cáscara de calabaza dentro del pozo, el otro saca el material hacia arriba con ayuda de una cáscara de calabaza atada a una soga. Para moverse dentro del pozo, se cavan en las paredes peldaños para los pies.

Bajo tierra trabajan sólo hombres. Las mujeres y los niños lo hacen en el exterior, extra- 


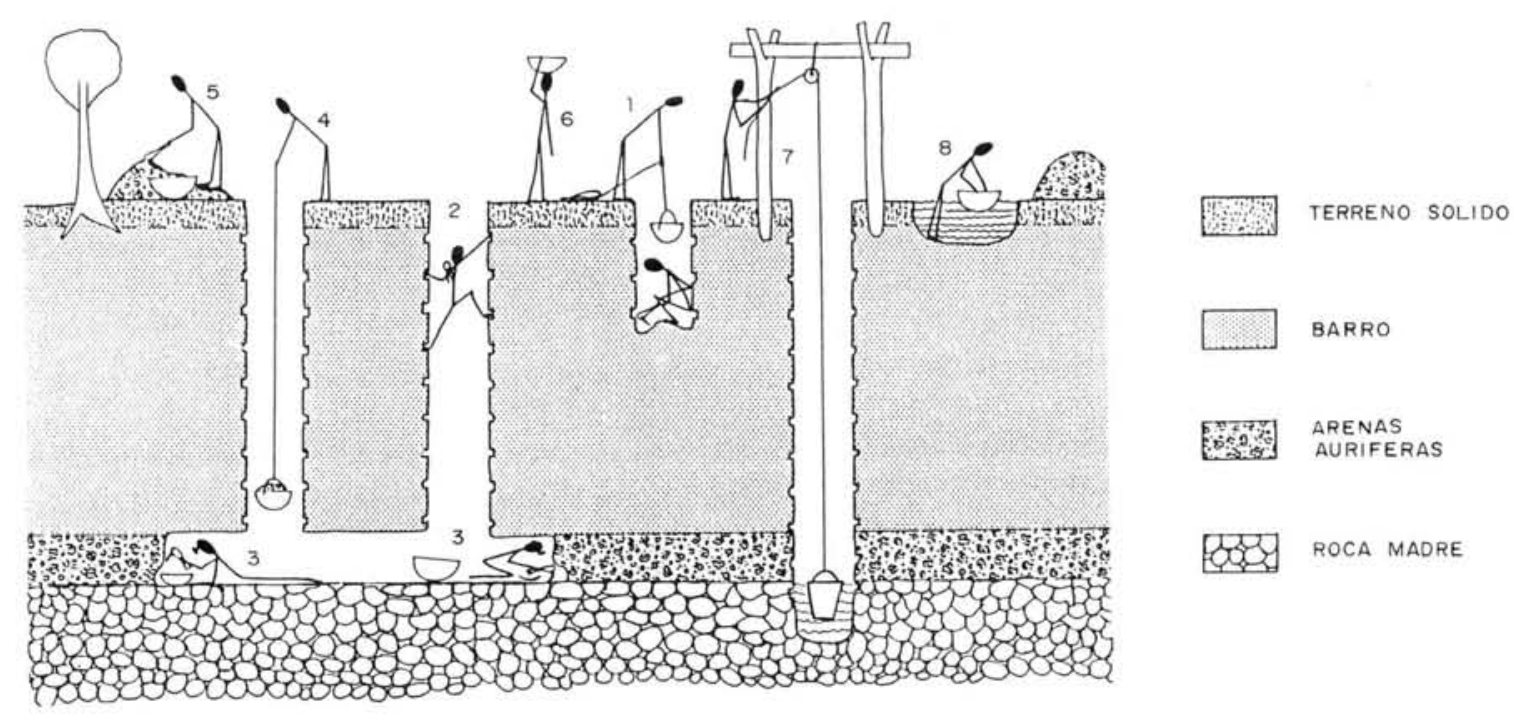

Fig. 5. Esquema de una mina de oro: 1) perforación y cavado del pozo; (2) movimiento en el pozo; (3) picado de la roca; (4) tracción funicular; (5) desmenuzado del material aurífero; (6) transporte sobre la cabeza; (7) elevación de agua; (8) lavado en pozas de agua con cáscaras de calabaza.

yendo la tierra y lavando el material que contiene oro con una cáscara de calabaza, mientras están de pie en un hoyo con agua hasta las rodillas. La pureza del oro extraído es del $94,80 \%$. Los mineros venden oro en polvo a comerciantes del oro, que revenden el metal precioso en el delta interior del Níger, en Bamaco, y a traficantes europeos. La mayor parte del oro de Malí está destinado a la exportación; solamente una parte insignificante se trabaja en los talleres de orfebrería locales. Sin embargo, a menudo, también antiguas joyas van a parar al crisol.

\section{JOYAS DE ORO DE MALI}

Más de veinte etnias viven en este estado interior del Africa Occidental en los márgenes meridionales del Sahara, nómadas o semisedentarias, ganaderos, pescadores y campesinos sedentarios. Cada grupo étnico tiene sus propios artesanos, entre los cuales orfebres y herreros pertenecen a una casta profesional endogámica. Correspondiendo con la tradición ornamental de su respectiva etnia, están especializados en determinados materiales y técnicas de fabricación.

T. P.. $\mathrm{n}^{\circ} 50.1993$
Según la forma de las joyas, es posible distinguir la pertenencia étnica de las mujeres, así como reconocer su estado civil y posición social. Son las mujeres de los fulbés, los songhay y los bozo las que llevan grandes pendientes de seccion cruciforme como parte de su vestimenta (Lám. I). Pueden llegar a pesar 400 gr. cada par. Los pesados pendientes, con hojuelas a modo de alas, no solo se sujetan en los lóbulos de la oreja, sino que también se sostienen con un hilo que pasa por la cabeza.

Los pequeños aros torsionados de sección cuadrada están muy extendidos y los llevan mujeres de muchas etnias. Al contrario que los aros de sección cruciforme que sólo aparecen como pendientes de orejas, aquéllos se llevan de modos diversos. Los aros prehistóricos comparables se interpretan a menudo como adorno para las orejas. En la multiplicidad de modos de llevarlos en Malí se hace evidente que también sean imaginables otras posibilidades, tales como adorno para la nariz, el cuello, el pelo o las sienes. En el tabique nasal se lleva un único anillo torsionado, o también a modo de dije en la frente o en el collar. En Malí pueden distinguirse hasta 20 aros para colgar del pabellón de la oreja. Como adorno entrelazado en artísticos peinados, su número no tiene límite (Lám. I). Además de estas joyas de oro, son muy aprecia- 

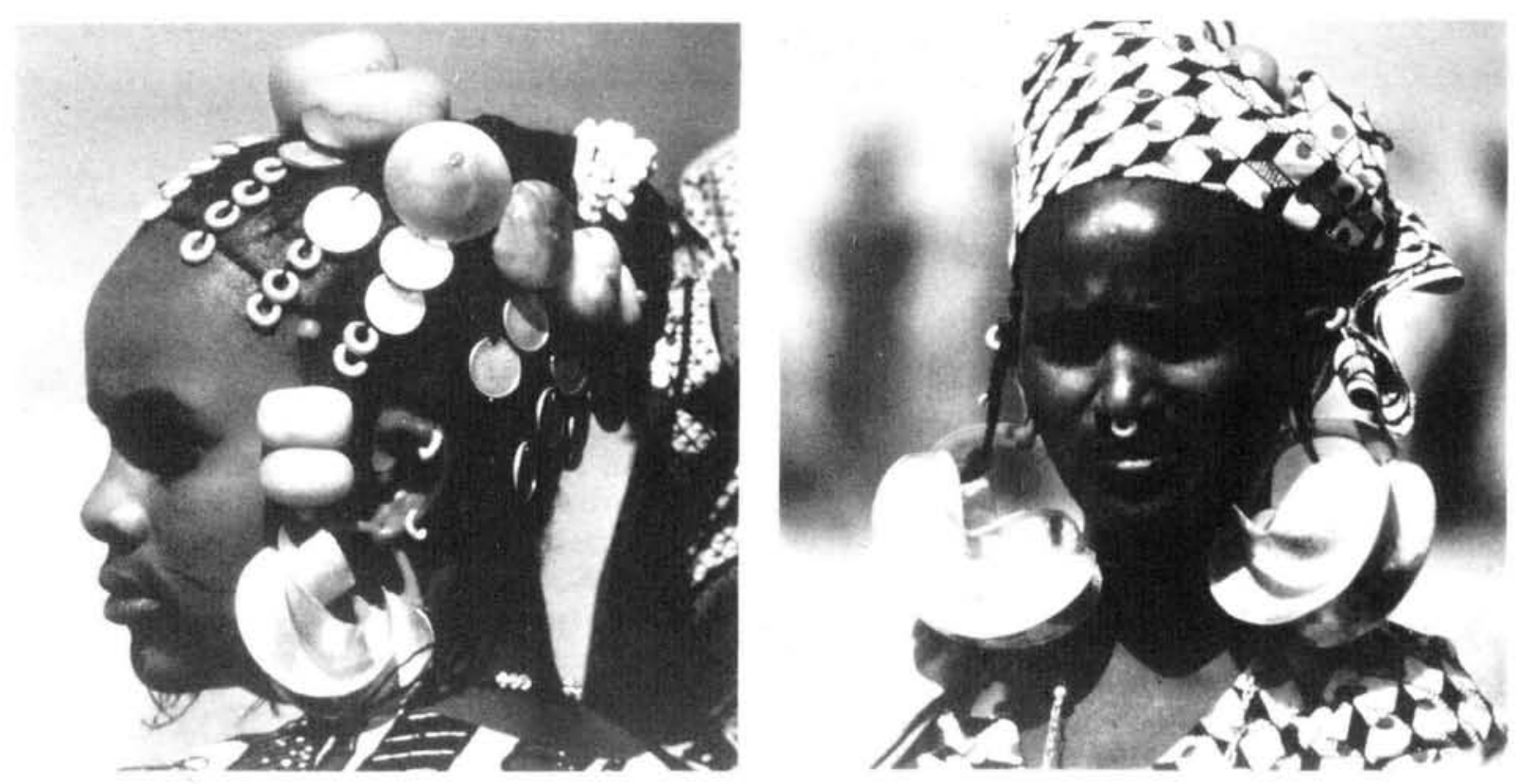

Lám. I. Mujeres fulbés ataviadas como Diafarabé. Mali. Cuentas de ámbar, monedas de plata, aros a partir de una barra doblada, en el pelo, nariz y orejas, así como grandes pendientes de lóbulos pronunciados.

das las de filigrana soldada en hilo y lámina. Muchos orfebres dominan también la técnica de soldadura. A menudo se llevan combinadas ambas formas de adorno, las soldadas y las martilladas. Otros componentes ornamentales son diferentes cadenas de plata o latón, collares de cuentas de cristal, de ámbar y monedas de plata. El tatuaje y el vestido se consideran también parte del ornamento de una persona.

En Malí, las mujeres fabrican imitaciones de los aros forjados y torsionados en arcilla, hilo y paja. La forma de la luna en cuarto creciente parece tener tal fuerza simbólica que las mujeres que no pueden permitirse una joya de metal llevan joyas tradicionales hechas en materiales no preciosos. Se fabrican también imitaciones de filigrana en paja. Han llegado a ser especialmente conocidas las réplicas producidas en el norte del país, elaboradas a partir de paja alheñada sobre un núcleo de cera de abeja (Lhote, 1946; Gabus, 1982: 302-317). Este adorno se denomina "oro de Timbuctu». De todos modos, más frecuentemente que las imitaciones de paja se llevan réplicas de "aros torsionados" de arcilla pintada de amarillo, de los cuales existe un modelo correspondiente a cada tipo.
Vistos desde una cierta distancia, las joyas de arcilla se asemejan mucho a los originales.

\section{EL TALLER DE ORFEBRE}

En Africa Occidental, los talleres se sitúan en diversos lugares: tiendas de campaña, casas o cabañas, en un patio interior, al borde de la carretera o en el mercado, en medio de una aldea o a las afueras. Existen además artesanos ambulantes que se establecen en un lugar al aire libre por un tiempo. Los conocimientos de la orfebrería se transmiten de padres a hijos, o a otros miembros masculinos de la familia. Las esposas de los orfebres trabajan a menudo como alfareras.

Donde mejor puede observarse la fabricación de los aros de oro torsionados es en el delta interior del Niger, en las ciudades de Djénné o Mopti (5) (Gardi, 1985: 270-297).

En el taller de orfebre, los puestos de trabajo se encuentran a ras de suelo (Fig. 6).

(5) Para la investigación se encargaron en 1987 varios tipos de aros de oro en el taller de Bamoye Yattara (Songhay), Mopti. El orfebre que lo realizó fue Seikou Konipo. 


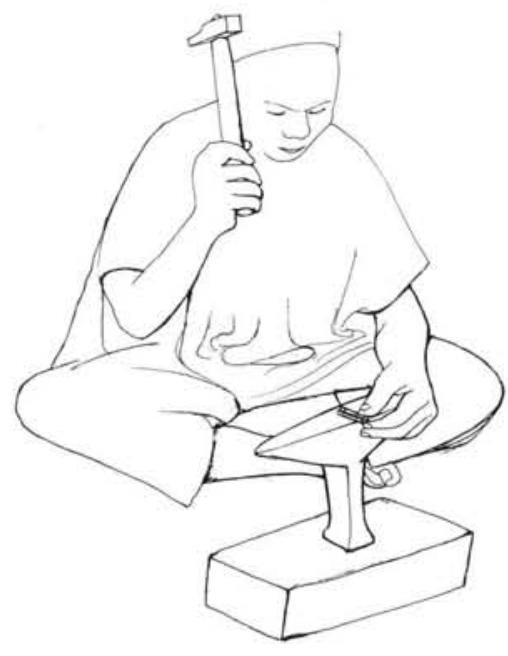

Fig. 6. Posición de trabajo para el forjado de la barra cuadrada.

Una esterilla o una piel sirven para sentarse. El yunque se introduce en una traviesa de madera, sujeta a su vez al suelo. El lugar central es el horno cavado en la tierra, con fuelles de saco, de tambor, o de ventilador (Cline, 1939: 103). El fuelle lo maneja el aprendiz. Para poner al rojo las piezas, además del horno, hay pequeños hornillos de carbón vegetal fabricados en alambre de hierro, atizados con un abanico.

Los crisoles de cerámica los hace el propio orfebre. El molde de vaciado para el lingote, fabricado localmente, se hace también de arcilla. Sin embargo, hoy se suelen utilizar moldes de hierro colado importados. Para fabricar los aros de oro tradicionales hay en el inventario de herramientas distintas tenazas, martillos, yunques, cinceles, punzones, buriles, palos y giramachos. Para la producción de la filigrana y el granulado hay además limas, una lámpara de soldar y un soplador. Si bien en muchos casos el orfebre está en condiciones de forjar sus propias herramientas, es, sin embargo, el herrero, quien fabrica habitualmente las herramientas del orfebre. Las piedras de afilar, las cenizas y la arena del suelo del taller sirven para alisar y pulir las joyas. Para el tratamiento de la superficie hay en cada taller varios cuencos con baños de tintura y limpieza. El orfebre necesita una piedra de toque de pizarra (Moore y Oddy, 1985) y una balanza de brazos, a fin de examinar los compo- nentes de la aleación del oro y pesar el material de partida y el producto final. El precio se calcula en función del peso de la aleación de metal precioso trabajado.

\section{FABRICACION DE AROS TORSIONADOS CON PERFIL CRUCIFORME (6)}

El material de partida para los pendientes de los fulbés es un lingote de oro de una aleación 750/000. En una cazuela de barro se funde oro y plata fina en una proporción de 3:1, y se vacía en una lingotera. Para un par de pendientes, del lingote se separa un trozo correspondiente de $33 \mathrm{gr}$. Con un pesado martillo se transforma el trozo en un prisma cuadrangular de sección cuadrada $(4 \times 4 \mathrm{~mm}$.) (Fig. 7a). El oro se trabaja en frío. Pero durante todo el proceso de transformar el lingote en joya (Figs. 7-9f) es preciso volver a recocer varias veces, a fin de hacer forjable el metal, que se vuelve duro y quebradizo al darle forma. Después de recocer, el metal se enfría en agua o en arena. Cuando el artesano olvida el recocido, aparecen grietas y la pieza tiene que ser fundida de nuevo. La secuencia de la transformación de la barra de sección cuadrada hasta el producto final se ve en la figura 7.

Para fabricar dos pendientes, se parte la barra de oro cuadrangular por el centro a fin de obtener dos piezas de igual peso, que luego se trabajan paralelamente. Después se acanalan los cuatro lados planos de la barra trazando con un cincel una estría de $1,7 \mathrm{~mm}$ aproximadamente a lo largo del centro de la cara, pero sin llegar a tocar los extremos (Fig. 8a). La sección tiene ahora forma de hoja de trébol (Fig. 7b).

El orfebre pone entonces la barra acanalada en horizontal, junto al borde del yunque, y quita con un martillo especial el material que encuentra entre las estrías hechas con el cicel (Figs. 7c-e y 8b). El martillo y el yunque forman un ángulo menor de $90^{\circ}$, para que el metal entre las estrías y las láminas pueda ser trabajado. Después se forja el metal en el borde del yunque (Fig. 9a). Durante este largo proceso hay que recocer varias veces. En cuanto las láminas han

(6) Una descripción detallada de la fabricación de aros torsionados de sección cuadrada o cruciforme se encuentra en Armbruster (1988: 58-86; 1993; 63-64). 

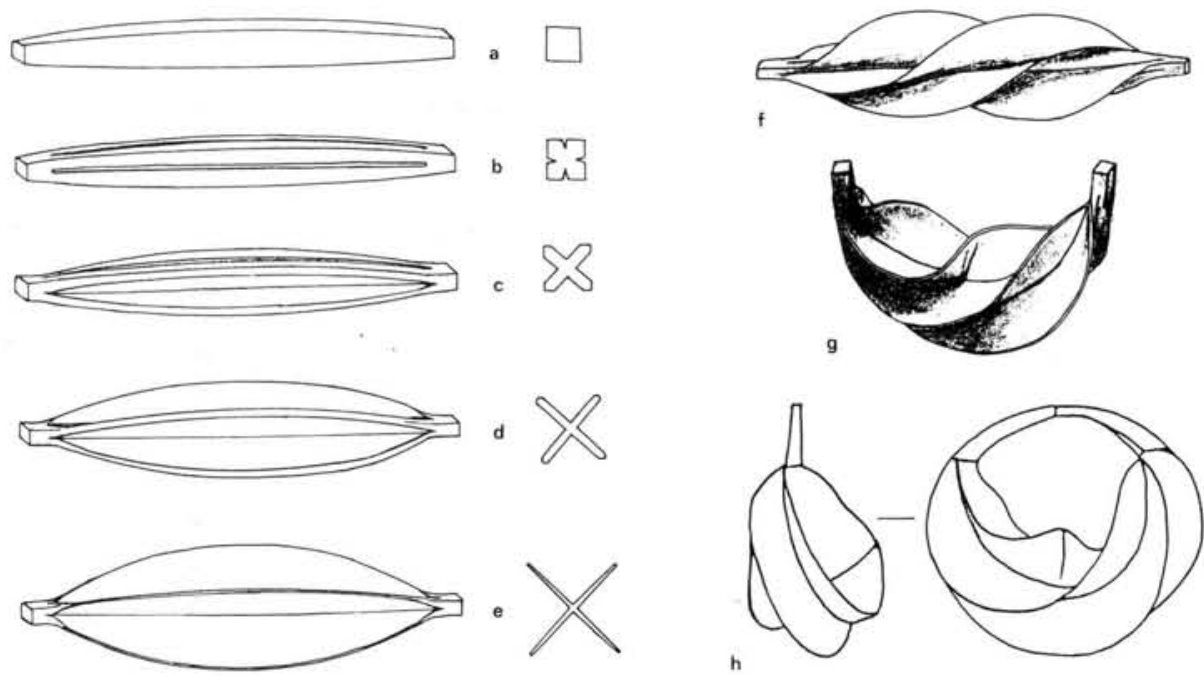

Fig. 7. Fabricación de aros de lóbulos pronunciados de sección cruciforme: a) barra cuadrada; b) estría hecha con el cincel; c) bordes rebajados; d) láminas rebajadas y forjadas; e) fase avanzada de forjado; f) torsionado alrededor del eje; g) forma de media luna; h) producto final.

alcanzado el tamaño previsto, se coge la pieza por sus extremos con dos tenazas y se gira alrededor de su eje central (Fig. 9b). Se pueden obtener así láminas en forma de ala. Con unas tenazas, un martillo redondo y un yunque de ángulos agudos se ponen derechas las láminas y se les da la forma deseada (Figs. 9c-d). Luego, el orfebre dobla la pieza hasta darle forma de media luna, aplastándola y extendiéndola. Después de que se hayan forjado los extremos en hilos cuadrangulares, para colgarlos de las orejas, y se hayan doblado en el yunque con un punzón redondo (Figs. 9e-f), el pendiente ha alcanzado su forma definitiva. A fin de dar a la joya una brillantez aún más especial, se trabajan los cantos de la lámina con pequeños golpes de cincel. Para ello, el pie sirve como superficie para golpear y se hace con el mango del martillo. Como se ha dado forma al oro sin arranque de virutas, no se pierde nada de material. El tratamiento de la superficie comienza por el lijado. Con la mano, se frota arena fina y humeda del suelo del taller. Con ayuda de una tira de paño, húmeda e impregnada de esta arena, se puede limar entre las láminas. Los dedos del pie y una mano mantienen tensa la tira, mientras la otra sujeta el pendiente para limar.

Por último, las joyas ya acabadas se decapan, se tiñen y se pesan. Para el decapaje en
Malí se utiliza una mezcla de sal de cocina, tamarindo y limón, con algo de agua. En el baño de tinte, se refina o enriquece primero la superficie con oro fino, y luego se tiñe de oro rojo con una disolución de sal de cocina que contiene oxido de hierro (cf. Thiam, 1950: 4546; Armbruster, 1989, 99-100, 186-189, figs. 103-110). El tiempo de trabajo para fabricar estos pendientes de $33 \mathrm{gr}$. es de 4 horas y media.

\section{AROS DE SECCION CUADRADA (Fig. 10)}

Los aros que se llevan como pendientes, en el cuello, el pelo o la nariz se hacen a partir de una barra cuadrangular, que puede haber sido estriada en la mitad de sus caras. Cada par de pendientes puede alcanzar de 10 gr. hasta 150 gr. o más. Para fabricar estos pesados y macizos aros, dos orfebres tienen que torsionar la resistente barra cuadrangular con dos giramachos aplicando mucha fuerza. Llamamos giramachos o terraja a dos placas oblongas de hierro con agujeros cuadrados (Armbruster, 1993: 63). Tras haber colocado y ajustado los giramachos en la sección de la barra, uno de los artesanos sujeta fuertemente el giramachos inferior en un tronco de madera, mientras el otro dobla el segundo. Entretanto, hay que recocer a menudo, ya que 

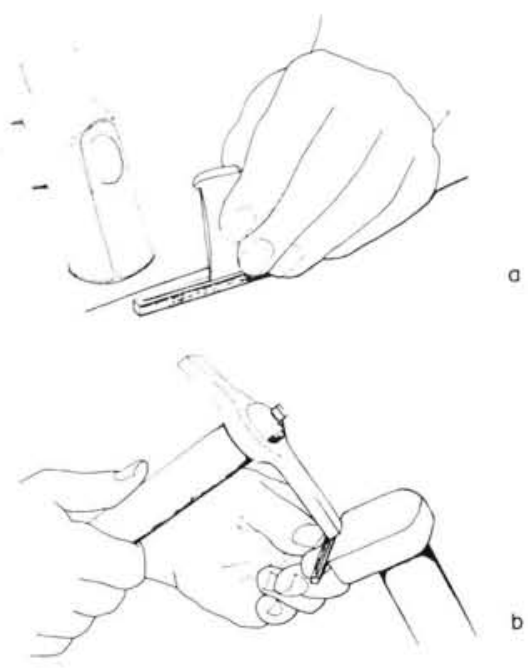

Fig. 8. a) Haciendo una muesca con el cincel; b) Retirando la moldura en el borde del yunque.

la barra se endurece enormemente por la deformación. Después de haber doblado y recocido alternativamente durante largo tiempo, la barra tiene un aspecto como de rosca. Se forjan luego los extremos, dándoles forma cónica y se golpea la barra con instrumentos de madera hasta obtener el aro deseado. En aros más pequeños no es necesario el giramachos para la torsión, ya que basta con las tenazas, sin hacer palanca. En este caso, la barra cuadrangular martillada se torsiona igualmente con ayuda de dos tenazas, o bien, se hace una estría con el cincel en el centro de los lados antes de la torsión. Después se hacen los extremos cónicos, y se le da a la barra recta forma de aro, doblándola y golpeándola con martillos de madera y metal sobre una base de madera (comparar con los ejemplares de Lisboa en la Fig. 3a-d).

\section{ETNOARQUEOLOGIA Y ANALOGIA EN EL AMBITO TECNICO-ARTESANAL}

"Much can be learned from the ethnologist's study of primitive tools... Hence the story of tools will have to be built up from the study of properly dated pieces from excavations and museums, and their handling can still be studied in primitive smithies, which prove to be well arranged and properly planned for the execution of the various operations of the primitive smith" (Forbes, 1964, VIII : 130).

Las coincidencias en el aspecto y fabricación de los actuales aros de oro del Africa Occidental y las joyas europeooccidentales de la Edad del Bronce son evidentes. Resulta natural, por tanto, hacer una comparación sobre una base tipológica y técnica. En cualquier caso, los trabajos de orfebrería morfológicamente comparables están tan alejados geográfica y cronológicamente que no se puede forzar ninguna conclusión sobre continuidad o divergencia. Estas técnicas pueden surgir independientemente en diferentes ámbitos temporales y geográficos.

La primera descripción diferente de las técnicas artesanales del Sudán Occidental fue una oportunidad para los arqueólogos de aprender, a partir de la etnografía, algo sobre los procedimientos artesanales, acentuándose las afinidades tipológicas de los aros torsionados de oro con los aros en espiral (Zeltner, 1915: 219). Ya a fines del siglo XIX y comienzos del XX (Evans, 1881: 393; Armstrong, 1920: 38) se compararon los aros de oro torsionados de la Edad del Bronce con aros de Africa. Ch. Eluère $(1980 / 81)$ señaló la afinidad tipológica y tecnológica de seis aros torsionados de Senegal con joyas de la Edad del Bronce. Estos aros senegaleses fueron a parar en 1886 a la colección del Musée des Antiquités Nationales, Saint-Germainen-Laye, donde están expuestos en la sala de arqueología comparada (catálogo Archéologie comparée, 1982: 45, n 84.050). Los pendientes de oro de barra torsionada de los depósitos franceses de Lanrivoaré, Departament Finistère (Eluère, 1982: fig. 171) y de Carcassone, Departament Aude (Eogan, 1967: fig. 7) son piezas que se pueden comparar exactamente con los aros de oro de Africa Occidental de sección cuadrada; los pendientes irlandeses de Castlereagh, Condado de Roscommon, con los de perfil cruciforme.

Encontramos a menudo curiosas afirmaciones sobre cuestiones de técnica artesanal en la prehistoria, lo cual hace evidente qué difícil resulta a los arqueólogos consultar con especialistas en estas cuestiones, y más aún hacerse ellos mismos expertos en ellas. Las hipótesis citadas más arriba sobre el método para la producción de los aros en espiral y de los 

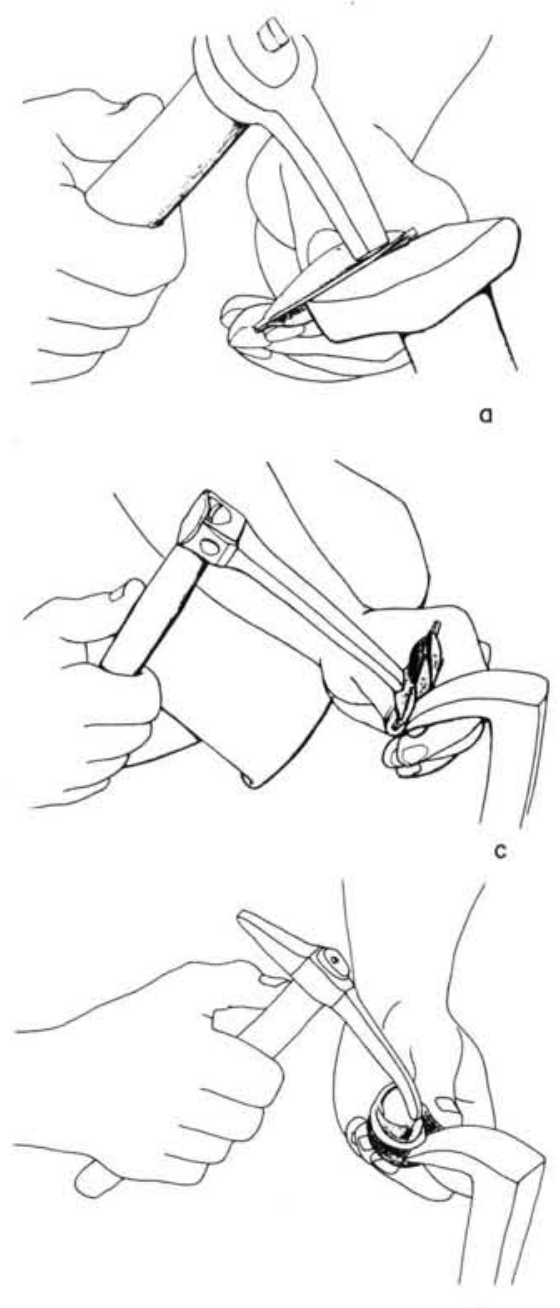

e

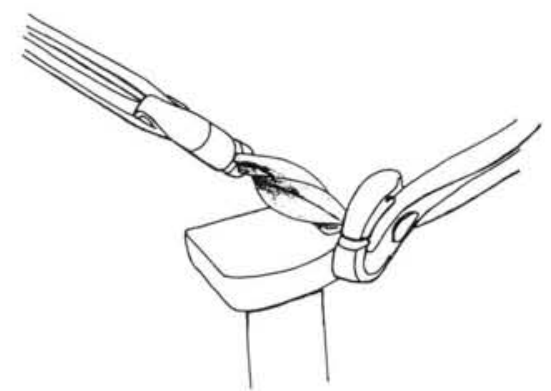

b

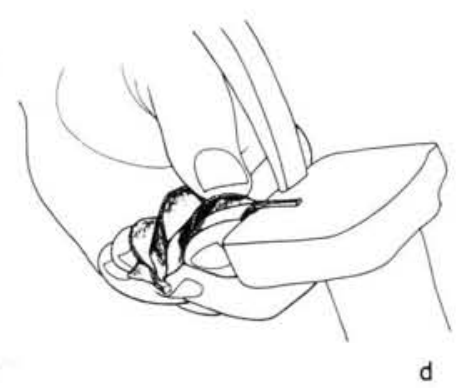

d

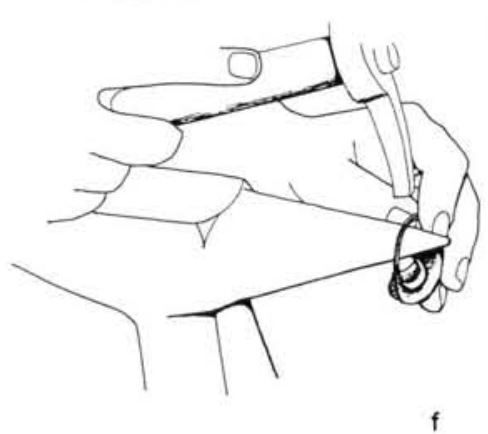

Fig. 9. a) Posición de las manos en el forjado de láminas. b) Torsionado alrededor del eje. c) Ajustado y forjado de las láminas en el pequeño yunque de ángulo agudo con martillo ligeramente redondeado. d) Forjado de los extremos ahuecados hasta conseguir hilos de sección cuadrada. e) Ajustado en el yunque pequeño. f) Acabado del enganche para colgar el pendiente.

torques ilustran la capacidad y límites de los arqueólogos en el tema del trabajo del metal.

Para reconstruir técnicas que hoy carecen de aplicación alguna entre nosotros, resulta inevitable hacer experimentos y/o buscar analogías funcionales como modelos explicativos. Estos deben examinarse críticamente y, si es posible, llevarse a efecto adaptándose a las correspondientes condiciones prehistóricas. Podemos enumerar formas de cultura material que, en apariencia, función y fabricación, siguen vivas hoy en nuestro entorno cultural, o que al menos están documentadas históricamente, y a partir de ellas referirnos al material arqueológico encontrado (Smolla, 1964: 31; Hodder, 1982: 12). Para describir artefactos prehistóricos que nos son desconocidos es preciso encontrar conceptos actuales. A menudo, el arqueólogo aplica, inconscientemente a veces, paralelos de trasfondo contemporáneo.

En su obra "The present past", Hodder hace una dura crítica a muchos de sus colegas 

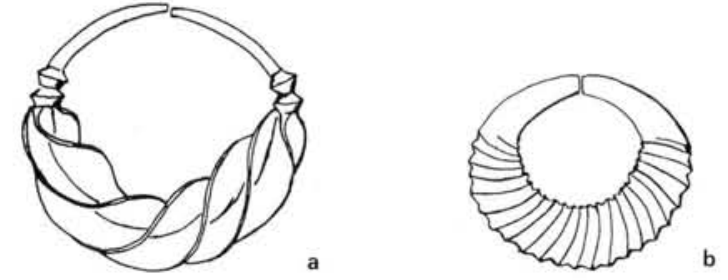

0 $3 \mathrm{~cm}$

Fig. 10. Mali, 1987: a) Pendiente de lóbulos pronunciados; b) Aro a partir de una barra doblada.

que trasladan características de las sociedades actuales a contextos prehistóricos, y considera por ello que esta analogía es aplicable sólo con reservas. Con los métodos tradicionales, el arqueólogo puede describir, ordenar comparativamente y diseñar ciclos y mapas de dispersión, pero el fondo y los procesos mentales siguen estando ocultos para él. Con ayuda de los métodos de las ciencias naturales, se pueden analizar los hallazgos, obteniendo resultados inequívocos. Los métodos de otras disciplinas ajenas ayudan a plantear modelos de interpretación y hacer más probable una posibilidad entre muchas (Strahm, 1985: 167).

Los* mejores resultados en relación con la arqueología experimental y la analogía funcional paræ̊ investigar las posibilidades y probabilidades del passado no transmitido por escrito se han alcanzado en el ámbito de la tecnología (Crawford, 1982: 6). Precisamente para los primeros trabajos en metal, de los que tenemos documentados muchos objetos pero pocas herramientas, pueden esperarse interesantes resultados. R. J. Forbes (1950), en "Metallurgy in Antiquity", toma de las ciencias naturales y de la etnología argumentos para la reconstrucción de la metalurgia más antigua. M. J. Rowlands (1971: 210221) enfatiza el valor de los datos etnográficos para un amplio espectro de interpretaciones posibles de la metalurgia prehistórica (sobre métodos de fabricación, obtención de la materia prima, organización, estatus, especialización, intercambios y migraciones).

Para las técnicas de fabricación de joyas se han propuesto reiteradamente analogías con talleres recientes. Así lo hace, por ejemplo, A. Goetze (1913) sobre la técnica de vaciado de las cadenas en bronce de la Edad de Bronce. Propone como modelo explicativo los moldes de vaciado actuales y el modo de fabricación de cadenas similares en Malasia, cuyos eslabones se vacían unos en otros. Como analogía para la fabricación de pulseras de vidrio celtas, M. Korfmann (1966) describió el trabajo de los artesanos en Hebron, Jordania, que fabrican todavía en la actualidad pulseras semejantes, dejando claro que además de la observación detallada de los métodos de fabricación de productos artesanales, también el mobiliario de los talleres, la organización, la tradición familiar, etc. pueden proporcionar importante información para el arqueólogo. Por el contrario, A. Pietzsch (1964: 9) constató: «Sin embargo, nadie puede estar hoy de nuestra parte cuando queremos juzgar — dotados de todos nuestros conocimientos técnicos y adelantos-el trabajo técnico de la antigüedad. Tenemos que revivirlo trabajando nosotros mismos, sin modelos, en las condiciones dadas".

La posibilidad de una colaboración entre diferentes disciplinas, como la prehistoria y etnología, para el estudio de estas cuestiones, se ha discutido vehementemente en los últimos años. También el desarrollo de la «New Archaeology" ha contribuído a formular nuevamente el concepto de analogía y a discutir sus posibilidades y límites (Eggert, 1978). Es en el conjunto terminológico de esta discusión donde hay que colocar conceptos como «arqueología experimental» (Coles, 1979; Experimentelle Archäologie, 1991; Devermann y Fansa, 1992), "etnoarqueología" (aclaración de concepto, con divergencias de interpretación, en Hodder, 1982: 28 ss y Smolla, 1988: 127 s.), «comparación actualista» (Ziegert, 1980: 56. 63 fig. 1) y «analogía” (Smolla, 1964: 30-35; Hodder, 1982: 11 ss). El diálogo sobre el significado y uso de analogías al plantear argumentaciones etnoarqueológicas ha sido tratado críticamente por A. Gould y P. J. Watson (1982: 355-400). El enfrentamiento sobre el concepto de analogía se debatió más tarde, en «La reacción contra la analogía" (Wylie, 1985: 63-107). Este debate es particularmente crítico con la trasposición directa de fenómenos sociales y económicos de un contexto etnológico para interpretar contextos prehistóricos.

En el marco de este estudio técnico, no se

T. P.. $\mathrm{n}^{\circ} 50.1993$ 
ha podido profundizar en cuestiones sociales ni económicas sobre la artesanía del oro o la posición del orfebre, ni tampoco en el contenido simbólico, la tradición e innovación de la producción de joyas. Estas cuestiones, sin embargo, son tan importantes como los aspectos técnicos del trabajo del metal. Los artefactos arqueológicos, ya se interpreten como joyas, útiles u objetos de uso corriente, llevan consigo numerosa información. Para interpretar una joya es preciso tratar también la cuestión de su origen. Así, debe identificarse también el material y las fuentes de materias primas, los métodos de extracción, así como el comercio de adquisición de metales, y cuestiones similares. En cuanto a la fabricación, es preciso conocer las herramientas que corresponden a la técnica empleada. Pero la ejecución de una joya no solo exige el material de partida y un taller equipado, sino, sobre todo, un artesano con los conocimientos de los tipos de joyas, las técnicas de trabajo y la destreza práctica. El aprendizaje de técnicas de orfebrería supone un artesanado organizado y consciente de su tradición. Los orfebres pueden trabajar permanentemente en un sitio si tienen encargos suficientes. Pero también pueden coger sus herramientas y, como artesanos ambulantes, propagar por amplios espacios geográficos sus conocimientos técnicos y tipológicos. También es posible que reciban nuevas influencias durante sus años de peregrinaje y las introduzcan luego en su región de origen.

La transmisión de formas depende de su contenido simbólico y la apreciación de su valor dentro de un entorno social. Las joyas no solo se llevan como un objeto de prestigio sino que informan sobre el estado civil, la edad y la pertenencia étnica. Hay que tener en cuenta que casi nunca se lleva un solo elemento ornamental. Es posible adornarse con una combinación de piezas diferentes, confeccionadas además con materiales distintos, y que se llevan en diferentes partes del cuerpo, en el pelo y en el vestido. El corte y el color del vestido, al igual que el peinado, forman parte también de lo ornamental.

La creación en los diversos ámbitos del artesanado, que en la prehistoria aún no se habían dividido entre arte y artesanía, tiene una relación directa con el contexto social. La continuidad en la tipología de las joyas está en relación con la continuidad y la cohesión de una sociedad tradicional. Además, para la innovación en el ámbito de las joyas, es preciso tener en cuenta factores como el cambio social. Una joya sólo se fabricará cuando lo permitan las condiciones económicas y el acceso a las fuentes de materia prima.

Esperemos que estas complejas cuestiones sobre artesanado sigan documentándose y analizándose en futuros trabajos.

\section{BIBLIOGRAFIA}

ANQUeTIL, J. (1977): "L'artisanat créateur au Mali». Paris. ARMBRUSTER, B. R. (1989): "Das traditionelle Goldschmiedehandwerk in Mali (Westafrika) als Analogie zu Beispielen bronzezeitlicher Metallverarbeitung". Unveröffentlichte Magisterarbeit am Institut für Vor-und Frühgeschichte der Universität Frankfurt/M.

- 1992 «Goldbergbau in Mali. Traditionelle Goldgewinnungsmethoden in Westafrika». Der Anschnitt, 3: 70-79.

- 1993 "Goldschmiedekunst in Mali (Westafrika)". Goldschmiede- und Uhrmacherjahrbuch: 52-73.

- (en preparación) "Os "brincos" aureos torcidos da collecçao do MNALV da Idade do Bronze "Atlántico". Um estudo etno-arqueológico". O Arqueólogo Portugues.

ARMBRUSTER, B. R. y S. DILlHÖFER (1988): «Das traditionelle Goldschmiedehandwerk in Bamako, Mali. Ein Vergleich von traditionellem und modernisiertem Handwerk $m$. ASAProgramm der Carl-Duisberg-Gesellschaft. Berlin.

ARMSTRONG, E. C. R. (1920): "Guide to the collections of Irish Antiquities: Catalogue of Irish gold ornaments in the collections of the Royal Irish Academy". Browne and Nolan, Ltd. Dublin.

BALDES, H. (1905): «Hügelgräber im Fürstentum Birkenfeld». Birkenfeld.

BRIARD, J. (1990): "Les influences d'Egée et du Proche Orient dans le Chalcolithique et l'Age du Bronze de la Bretagne». Orientalisch-ägäische Einflüsse in der europäischen Bronzezeit. Kolloquium Mainz 1985: 124-136. Bonn.

BróCKMANN, W. (1914): «Über die Herstellung der Wendelringe». Römisch-Germanisches Korrespondenzblatt 7 (3): 33-36.

BRUNN, W. A. (1953): «Ein Wendelringpaar aus Altmark». Altmärkisches Museum Stendal 7: 5-14.

CATÁlogo Colonia (1983): "Irische Kunst aus drei Jahrtausenden". Römisch-Germanisches Museum und WallrafRichartz Museum Köln. Köln.

CLINE, W. (1937): "Mining and metallurgy in negro Africa". Menasha, Wis.

Cohausen, C. A. (1985 y 1986): «Der Wendelring». Analen des Vereins für nassauische Altertumskunde und Geschichtsforschung, 19: 176-177.

Coles, J. N. (1979): "Experimental archaeology". London, New York. 
CoutIL, L. (1912): «Enclumes de l'Age du Bronze», L'homme préhistorique, 10: 97-104.

CRAWFORD, H. (1982): “Analogies, anomalies and research strategy". Paleoorient, 8 (1); 5-11.

DEVERMANN, H. y FANSA, M. (1992): «Bibliographie zur Experimentellen Archäologie». Archäologische Mitteilungen aus Nordwestdeutschland, Beiheft 7. Oldenburg.

EGGERT, M. K. H. (1978): «Prähistorische Archäologie und Ethnologie. Studien zur amerikanischen New Archaeology». Prähistorische Zeitschrift, 53: 6-164.

EHRENBERG, M. R. (1981): "The anvils of Bronze Age Europe». The Antiquaries Journal, 61 (1): 14-28.

ELÙERE, Ch. (1980 y 1981): «Reflexion à propos de "boucles d'oreilles" torsadées en or de types connus à l'Age du Bronze». Antiquités Nationales 12/13: 34-39.

- 1982: “Les ors préhistoriques». Coll. L'Age du Bronze en France, 2. Paris. 1982.

EogAN, G. (1964): "The Later Bronze Age in Ireland in the light of recent research". Proceedings of the Prehistoric Society, 30: 268-351.

- (1967): "The associated finds of gold bar torcs". Royal Society of Antiquaries of Ireland, 97 (1): 129-175.

- (1983): "The hoards of the Irish Later Bronze Age". University College Dublin.

- (1990): «Possible connections between Britain and Ireland and the East Mediterranean Region during the Bronze Age». Orientalisch-ägäische Einflüsse in der Europäischen Bronzezeit. Kolloquium Mainz 1985: 155-163. Bonn.

Evans, J. (1881): "The ancient Bronze Implements of Great Britain and Ireland". London.

"Experimentelle Archäologie Bilanz 1991" (1991): Archäologische Mitteilungen aus Nordwestdeutschland, Beiheft 6. Oldenburg.

Forbes, R. J. (1950): "Metallurgy in Antiquity». Leiden.

- (1963 y 1964): "Studies in ancient technology". 7/8. J. Brill. Leiden.

GABUS, J. (1982): «Sahara, bijoux et techniques». Neuchatel.

GARDI, B. (1985): «Ein Markt wie Mopti. Handwerkerkasten und traditionelle Techniken in Mali». Basler Beiträge zur Ethnologie. Basel.

- (1988): "Mali, Land im Sahel». Begleitheft zur Ausstellung. Basel.

GoetZe, A. (1913): “Die Technik gegossener Bronzeketten» Monteliusfestschrift. Stockholm: 155-175.

- (1925): «Bronzetechnik», In «Ebert Reallexikon der Vorgeschichte", II: 170-179, Berlin.

Gould, R. A. y WATSON, P. J. (1982): “A dialogue on the meaning and use of analogy in ethnoarchaeological reasoning». Journal of Anthropological Archaeology, 1: 355-382.

KIÉTHÉGA, J. B. (1983): “L'or de la Volta Noire. Explotation traditionelle, histoire et archeologie (Region de Poura. Haute-Volta». Paris.

Korfmann, M. (1966): «Zur Herstellung nahtloser Glasringe». Bonner Jahrbuch: 48-62.

HAWKES C. (1961): "Goldearrings of the Bronze Age, east and west". Folklore, 72: 438-474.

HEYNOWSKI, R. (1992): «Eisenzeitlicher Trachtschmuck der Mittelgebirgszone zwischen Rhein und Thüringer Becken". Archäologische Schriften Bd. 1. Mainz.
Hodder, I. (1982): "The present past: an introduction to Anthropology for archaeologist. Batsford. London.

JOCKENHÖVEL, A. (1982): «Zu den ältesten Tüllenhämmern aus Bronze». Germania, 60 (2): 459-467,

KRONE, O. (1937): «Wir schmieden einen germanischen Wendelring". Germanenerbe: 219-221.

LEVTZION, N. (1981): "Corpus of early arabic sources for West African history". Cambridge.

Lhote, H. (1946): «Bijoux en Paille de Tombouctou». Notes africaines, 32: 4-8.

MARYON, H. (1936): "Soldering and welding in the Bronze and Early Iron Agen. Harvard Univ. Cambridge.

MOHEN, J. P. (1984 y 1985): "Les outils des métallurgistes de l'Age du Bronze en France" Antiquitées Nationales, 16/17: 89-96.

MoOre, P. T. y OddY, W. A. (1985): «Touchstones: Some aspects of their nomenclature, petrography and provenance». Journal of Archaeological Science, 12: 59-80.

Nicolardot, J. P. y Gaucher, G. (1975): "Typologie des objets de l'Age du Bronze en France. Fasc. 5: Outilsm. Societé Préhistorique Française, Commission du Bronze. Paris.

OHLHAVER, H. (1939): “Der germanische Schmied und sein Werkzeug». Hamburger Schriften zur Vorgeschichte und germanischen Frühgeschichte 2. Leipzig.

OlDEBERG, A. (1942 y 1943): "Metallteknik under förhistorisk tid». Lund Bd., 1, 1942; Bd. 2, 1943.

PARREIRA, R. y PINTO, C. V. (1980): “Tesouros da arqueologia portuguesa no Museu Nacional de Arqueologia e Etnologia". Lisboa.

PietzSCH, A. (1964): "Zur Technik der Wendelringe». Arbeitsund Forschungsberichte zur sächsischen Bodendenkmalpflege, Beiheft 4. Berlin.

RowLANDS, M. J. (1971): “The archaeological interpretation of prehistoric metalworking». World Archaeology, 3 (2): 210-223.

Smolla, G. (1964): "Analogien und Polaritäten». Studien aus Alteuropa [Festschrift Tackeberg]: 30-36.

- 1988 «Ethno-Archäologie». In W. Hirschberg (ed.): "Neues Wörterbuch der Völkerkunde". Berlin: 127-128.

STRAHM, C. (1985) «Die naturwissenschaftlichen Methoden der Urgeschichtsforschung". In "Der Keltenfürst von Hochdorf. Methoden und Ergebnisse der Landesarchäologie" (Ausstellungskatalog). Stuttgart: 164-169.

TAYLOR, J. (1980) "Bronze Age goldwork on the British Isles». London.

THIAM, B. (1950): "Coloration des bijoux ouolof en or de basse qualité». Notes africaines, 46: 45-46.

TYLECOTE, R. F. (1986): "The prehistory of metallurgy in the British Isles". London.

UNTRACHT, O. (1982): "Jewelry, concepts and technology". London.

WYLIE, A. (1985): “The reaction against analogy». In M. B. Schiffer (ed.): "Advances in Archaeological Method and Theory". 8: 63-111.

ZELTNER, F. (1915): “Notes sur quelques industries du Soudan français". L'Anthropologie, 26: 219-234.

- (1931): "La bijouterie indigene en Afrique occidentale". Journal de la Société des Africanistes: 43-48.

ZIEGERT, H. (1980): “Objektorientierte und problemorientierte Forschungsansätze in der Archäologie». Hephaistos, 2: 53-67. 